\title{
High Capacity Image Barcodes Using Color Separability
}

\author{
Orhan Bulan, Basak Oztan, and Gaurav Sharma \\ University of Rochester, Rochester, NY, USA
}

\begin{abstract}
Two-dimensional barcodes are widely used for encoding data in printed documents. In a number of applications, the visual appearance of the barcode constitutes a fundamental restriction. In this paper, we propose high capacity color image barcodes that encode data in an image while preserving its basic appearance. Our method aims at high embedding rates and sacrifices image fidelity in favor of embedding robustness in regions where these two goals conflict with each other. The method operates by utilizing cyan, magenta, and yellow printing channels with elongated dots whose orientations are modulated in order to encode the data. At the receiver, by using the complementary sensor channels to estimate the colorant channels, data is extracted in each individual colorant channel. In order to recover from errors introduced in the channel, error correction coding is employed. Our simulation and experimental results indicate that the proposed method can achieve high encoding rates while preserving the appearance of the base image.
\end{abstract}

Keywords: c olor image barcodes, halftone-dot orientation, color separation

\section{INTRODUCTION}

Two-dimensional (2-D) barcodes are commonly utilized for carrying information in hardcopy documents addition to being cheap and convenient for automatic data retrieval, they offer higher capacity for data embedding than one-dimensional counterparts. Traditionally, these barcodes have been used in monochrome formats. ${ }^{1,2}$ However, more recently a number of color versions have been developed with a view to offering capacity and aesthetic improvements over the monochrome versions. ${ }^{3-8}$ The additional information capacity provided by two-dimensional barcodes enables a variety of applications such as carrying a digital signature that is used to verify the integrity of a document or carrying auxiliary data (e.g. biometrics) to authenticate the connection between identification cards (IDs) and card holders.

The fundamental drawback of traditional 2-D barcodes is their visual appearance. Since these methods embed data in a region that is solely dedicated to convey the information, the visual appearance of the encoded region is not a concern. For several applications (e.g. embedding data in personal IDs, driving licenses or credit cards), there is little or no flexibility for reserving an area for a barcode due to limited space. In these applications, data must be embedded in the existing document content. Conventional hardcopy watermarking techniques embed data in the print content unobtrusively without requiring additional space. ${ }^{9-11}$ But the payload data rates offered by these methods is significantly lower than that of 2 -D barcodes (often $1 / 10^{\text {th }}$ ) since the primary concern in these techniques is the visual quality of the primary image. Therefore, methods enabling high information capacity of 2-D barcodes while preserving the visual appearance of the print content are also desirable for addressing applications where the high data rate is the primary objective and aesthetics are a secondary, though still significant, consideration. Barcodes for product labeling, in particular, represent one such application scenario.

In this paper, we propose high capacity image barcodes that have ability to carry information in three color channels (cyan, magenta and yellow) of a base image. In particular, we encode data in each channel by utilizing

Send correspondence to O. Bulan: E-mail: bulan@ece.rochester.edu, Telephone: 1585 275-8122, Address: Electrical and Computer Engineering Department, University of Rochester, Rochester, NY, 14627-0126, USA, WWW: www.ece.rochester.edu/projects/iplab

This work was supported in part by the Air Force Office of Scientific Research (AFOSR) under grant number FA9550-071-0017, and by a matching grant from New York State Office of Science, Technology and Academic Research (NYSTAR) through the Center for Electronic Imaging Systems (CEIS). B. Oztan is now with the Rensselaer Polytechnic Institute, Troy, NY, USA.

Color Imaging XVI: Displaying, Processing, Hardcopy, and Applications, edited by Reiner Eschbach, Gabriel G. Marcu, Alessandro Rizzi, Proc. of SPIE-IS\&T Electronic Imaging, SPIE Vol. 7866 78660N - (c) 2011 SPIE-IS\&T · CCC code: 0277-786X/11/\$18 doi: 10.1117/12.872215 
dot orientation modulation motivated by our recently proposed hardcopy watermarking technique. ${ }^{10,11}$ Our strategy here is diametrically opposed to the one adopted in our recent work on watermarking ${ }^{10,11}$ : at the data encoding stage, we sacrifice image fidelity in favor of embedding robustness in regions where these two goals conflict with each other*. In order to extract the information in each color channel, we exploit the complementary relation between cyan, magenta and yellow (CMY) spaces and red, green and blue (RGB) colorants. We also employ error correction coding to identify the error free operational rates of our scheme. Our simulation and experimental results show that our method can achieve high encoding rates while still maintaining reasonable image quality.

In the literature, several other techniques have also been proposed for visually pleasing 2-D barcodes. The majority of these are however, applicable only to single channel images and offer significantly lower capacity than the proposed method. Shaked et al. ${ }^{12}$ propose visually significant barcodes (VSB) for bi-level images. These barcodes have an appearance of a computer logo or graphical indicia and are used to verify the integrity of the logo or indicium. Damera-Venkata et al. ${ }^{13}$ propose image barcodes (IB) by generalizing VSB to photographic continuous tone images. Both of these techniques do not exploit the flexibility for data encoding in a color image and hence, can only constitute a middle ground between traditional 2-D barcodes and hardcopy watermarking techniques in terms of embedding rates. In the color instantiation of DataGlyphs, ${ }^{14}$ data is encoded based on a color image by simply using the same glyph pattern for each separation in a dot-on-dot mode, inherently offering the same capacity as a single channel. All these techniques aim at high embedding rates, in addition, for their application scenarios, recognition of the base image by a human observer is typically sufficient. The proposed method offers a higher capacity and inherits several superior global and local synchronization properties from its related watermarking method. ${ }^{10,11}$

\section{SYSTEM OVERVIEW}

Figure 1 illustrates an overview of the overall proposed scheme. Here, m denotes message bits to be encoded and $I_{C M Y}(x, y)$ denotes a color base image that is utilized in the data encoding stage. In order to allow error recovery at the barcode decoder, the channel encoder first codes the message bits and produces the coded data c. The coded data $\mathbf{c}$ is then embedded in the base image during the halftoning process that is used to render the base image into a per-colorant bi-level image suitable for printing. The halftoning process jointly performs halftoning and data embedding for each of the C, M, and Y channels of the base image, generating a clustered-dot halftone $^{15}$ representation for each channel that uses elliptically shaped dots and incorporates the coded data as modulation of the orientation of the halftone dots. In the encoding stage, embedding robustness is emphasized over fidelity to minimize interference from the base image. The resulting barcode $I_{C M Y}^{b}(x, y)$ is then printed. The barcode decoder uses a a scanned version $I_{R G B}^{S}(x, y)$ as its input. From the scanned barcode, first C, M, and $\mathrm{Y}$ channels of the barcode are estimated and geometric distortions are compensated in each colorant channel. Then detection statistics in the form of moments $\sigma_{x}, \sigma_{y}$ are extracted from the halftone dots within each of the cells determined via the synchronization process. The extracted moments are then utilized in the channel decoder to estimate the transmitted message.

\section{COLOR IMAGE BARCODES}

\subsection{Orientation Modulation Based Data Encoding}

Color printing systems are typically based on multiple separations corresponding to cyan, magenta, yellow, and black colorants. These separations are first converted into bi-level images (typically via halftoning) and then printed in overlay to produce the (color) print. In order to facilitate embedding and detection on a per-channel basis we restrict ourselves to 3 -colorant CMY printing. In this case, we encode data in individual C, M, and Y channels of the base image as shown in Fig. 2.

In each colorant channel, we perform the modulation by utilizing dot orientation modulation motivated by our recently proposed hardcopy data hiding technique. ${ }^{10,11}$ Specifically, the method has ability to generate elliptically shaped halftone dots and provides flexibility to orient the dots along vertical and horizontal directions.

\footnotetext{
${ }^{*}$ These regions correspond to shadows, highlights, and highly textured image regions. ${ }^{10,11}$
} 


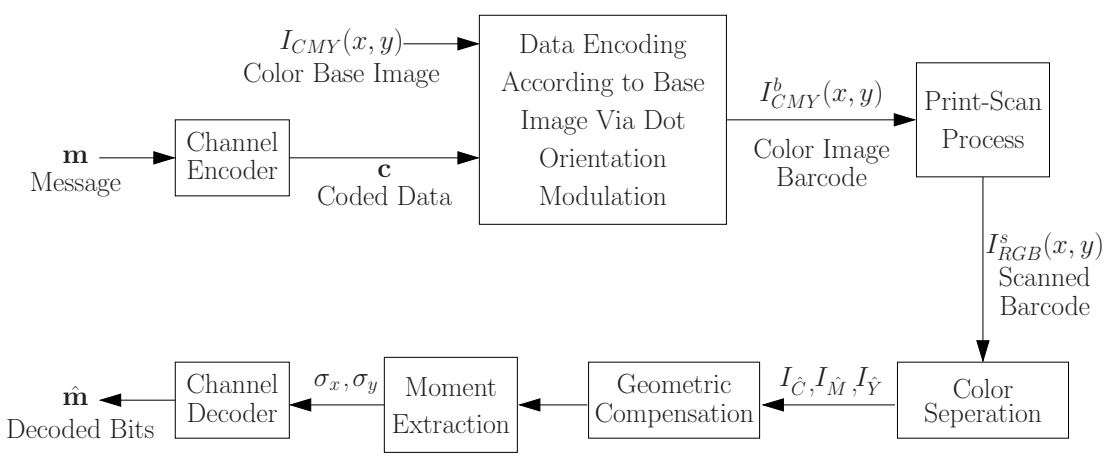

Figure 1. Block diagram for color image barcodes via dot orientation modulation and color separation.

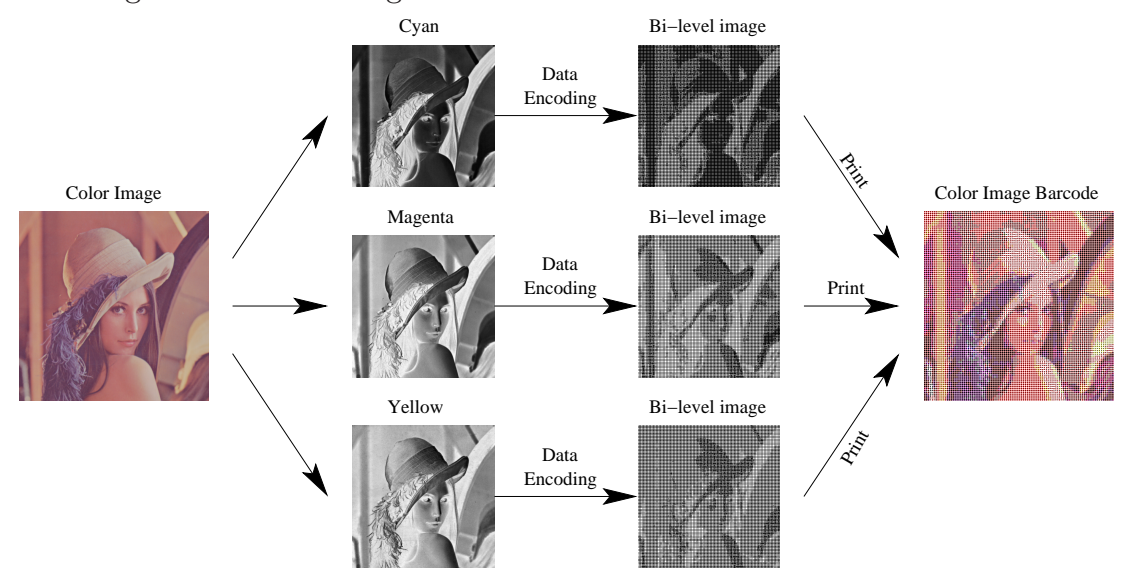

Figure 2. Per-colorant channel based data encoding. Scaling may impact the appearance of the bi-level images, which can be better examined by looking at the original electronic images at sufficient magnification..

Data is then embedded in the particular choice of the dot orientation. For this purpose, in our recent work ${ }^{10,11}$ we develop an analytical threshold function $T(x, y)$ that allows us to generate elliptically shaped halftone dots by comparing the continuous-tone image with the threshold function. As is the case for many threshold screens for clustered dot halftones, this threshold function offers no capacity for data embedding at midtones $(50 \%$ area coverage) to produce high quality prints. In order to achieve higher embedding rates, here we modify the threshold function in a way that it provides flexibility to encode data at midtones at a cost of lower quality for the printed base image. The modified threshold function is given by ${ }^{6}$ :

$$
T_{M}(x, y)=\frac{\left(1+\cos \left(2 \pi f_{x} x\right)\right)^{\gamma_{x}}\left(1+\cos \left(2 \pi f_{y} y\right)\right)^{\gamma_{y}}}{2^{\gamma_{x}+\gamma_{y}}}
$$

where $f_{x}$ and $f_{y}$ represent the frequencies along the horizontal axis $x$ and vertical axis $y$, respectively. And $\gamma_{x}$ and $\gamma_{y}$ are the design parameters that control the dot shape. The gain obtained for data embedding at midtones by using the modified threshold function is demonstrated in Figs. 3 (a)-(c). Figure 3 (a) illustrates a ramp that shows different shades of gray from white to black, which in the halftone print results in area coverage ranging from no colorant coverage (white) to full colorant coverage (black). Figure 3 (b) shows the corresponding bi-level image generated by comparing the ramp function with the threshold function $T(x, y)$ proposed in our recent work. ${ }^{10,11}$ The bi-level image gives an illusion of the continuous-tone ramp function and carries the data (all 0 's) in friendly regions ${ }^{\dagger}$. At midtones, however, it assumed a checker board format with no distinction between the vertical and horizontal orientations, which offers no capability for data embedding. Figure 3 (c) shows the

\footnotetext{
${ }^{\dagger}$ Friendly regions correspond to smooth regions between highlights (close to white regions) and midtones, and midtones and shadows (close to black regions). ${ }^{10,11}$
} 
(a) Contone ramp function

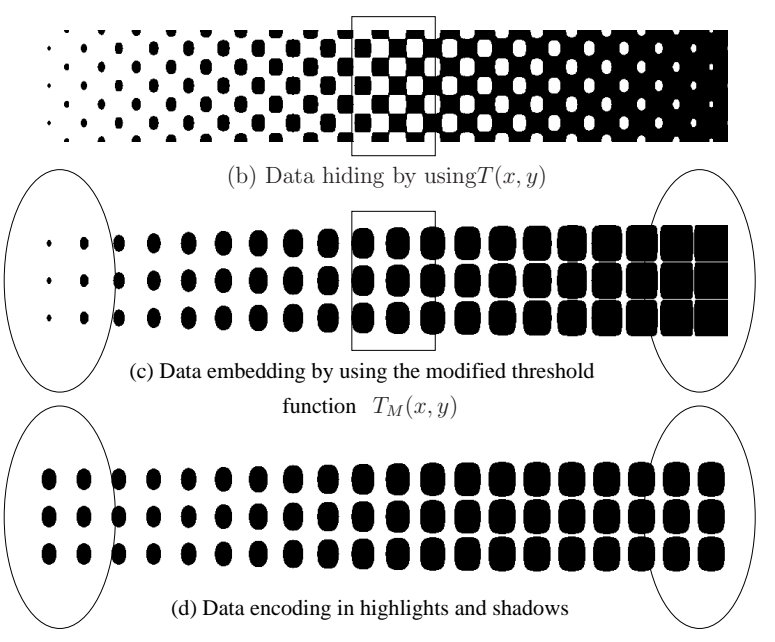

Figure 3. Data encoding via orientation modulation over various local graylevels.

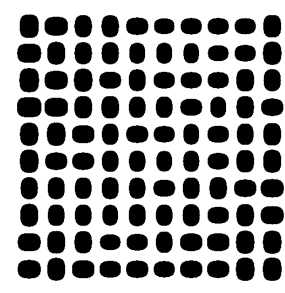

(a)

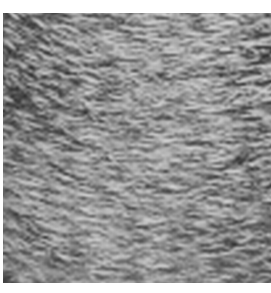

(b)

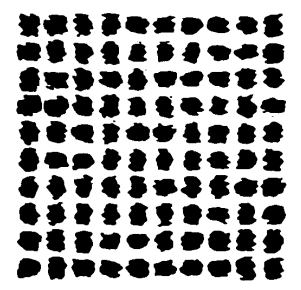

(c)

Figure 4. Data encoding via orientation modulation in highly textured regions of a base image: a) Dot orientation modulation based data encoding b) Highly textured region of a base image c) Dot orientation modulation based data hiding

binary image generated by using the modified threshold function $T_{M}(x, y)$. Though the binary image carries the data at midtones, in highlights (regions close to white) and shadows (regions close to black) the dot orientation is not identifiable because preserving the close to white (or black) image level leaves inadequate freedom for change in orientation. In these regions, we sacrifice the fidelity requirements in favor of embedding robustness and generate elliptical dots with identifiable orientation as shown in Fig. 3 (d); essentially compromising on the dynamic range to improve detectability of the data. In the highly textured regions of the base image, where fidelity requirements conflict with the embedding robustness, we similarly trade off image quality in favor of improved data detectability. In these regions, comparing the base image with the threshold function $T_{M}(x, y)$ results in loss of embedded data in favor of preserving the image content (Fig. 4 (c)). In order to achieve better detection performance at the barcode decoder, we again sacrifice the requirement of maintaining fidelity to the base image (Fig. 4 (a)) by replacing image values in each cell by the average over the cell, which approximated the template dot form of halftones ${ }^{16}$ used in the early days of digital halftoning. Figure 5 illustrates the overall data encoding process for a single colorant channel; the same methodology is used for all colorant channels.

\subsection{Color Separation}

At the barcode decoder, embedded data in each color channel $\mathrm{C}, \mathrm{M}$, and $\mathrm{Y}$ of the base image is extracted from a scan of the printed barcode. Unlike the printing systems, capture devices such as digital cameras or scanners utilize red, green, and blue spectrally sensitive channels to capture color images. Thus, C, M, and Y channels 


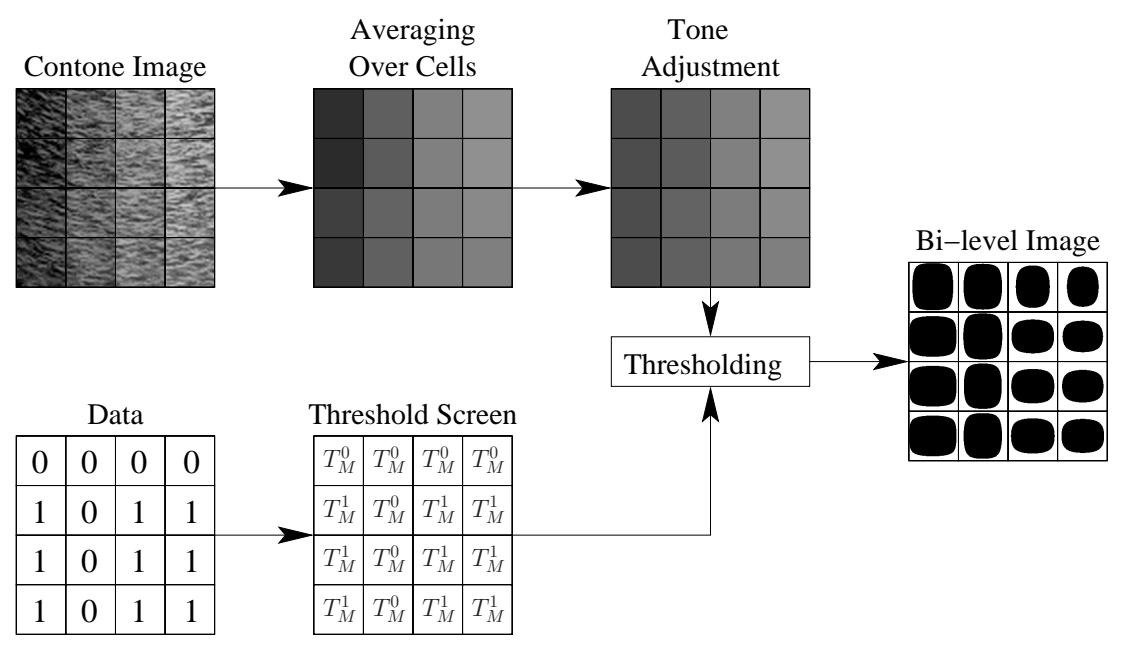

Figure 5. Per-colorant data encoding methodology.

of the color image barcode $I_{C M Y}^{b}(x, y)$ must be estimated from the scanned R, G, and B channels. In order to achieve this, we exploit the complementary relation between CMY and RGB colorants. Specifically, in an idealized printing and scanning system red channel only captures the information in cyan colorant, green in magenta and blue in yellow. In this idealized scenario, CMY colorants can be exactly obtained from the RGB channels, respectively. In practice, however, due to unwanted absorptions in the colorants, ${ }^{17,18}$ color channels interfere with each other. Estimates of the $\mathrm{C}, \mathrm{M}$, and $\mathrm{Y}$ colorant halftone channels obtained from the individual R, G, and B channels, respectively, include interference from other colorant channels. This interference affects the detection performance and causes errors. While the interference can be partly modeled and mitigated, ${ }^{6}$ in the present work, we do not address this and resort to error correction coding to recover from errors caused by interference and other sources of noise.

\subsection{Data Extraction and Error Correction Coding}

After the color separation, we first compensate geometric distortions in each colorant channel by utilizing the dot periodicity for global geometric distortions and decision directed synchronization algorithm for local distortions. ${ }^{6,10,11}$ From the synchronized individual colorant channels, orientation is detected by utilizing two orthogonal moments (i.e. each capturing the information along vertical or horizontal direction). From the estimated cyan channel of the image barcode $I_{\hat{C}}(x, y)$, the moment along $x$-axis within a cell $A$ is calculated as:

$$
\sigma_{x}=\frac{1}{U} \sum_{x, y \in A} I_{\hat{C}}(x, y)(x-\bar{x})^{2}
$$

where $U=\sum_{x, y \in A} I_{\hat{C}}(x, y)$ and $\bar{x}=\frac{1}{U} \sum_{x, y \in A} I_{\hat{C}}(x, y) x$ represents the abscissa of the center of mass of the dot. In each colorant channel, the moment $\sigma_{y}$ along vertical direction can be calculated similarly. After calculating the moments in each cell, data can be extracted by comparing the difference between the moments $\sigma_{x}-\sigma_{y}$ and a threshold value $t$. In order to better capture the information represented by the moments, we use a three level detector that either estimates the embedded bit value as a $0 / 1$ or declares an erasure. This is illustrated in Fig. 6.

Due to the print scan distortions and interference between individual colorant channels, the extracted data have some errors and erasures. In order to realize a practical system we employ error correction coding by exploiting the redundancy introduced at the channel encoder. In particular, we utilize repeat accumulate (RA) codes $^{19}$ motivated by the fact that they operate near capacity for binary erasure channels. The message recovery from the scanned barcode is illustrated in Fig. 7. 


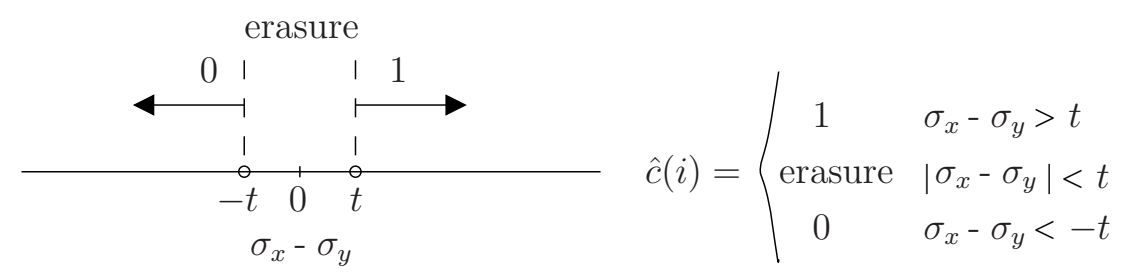

Figure 6. Estimation of embedded data based on the extracted moments.

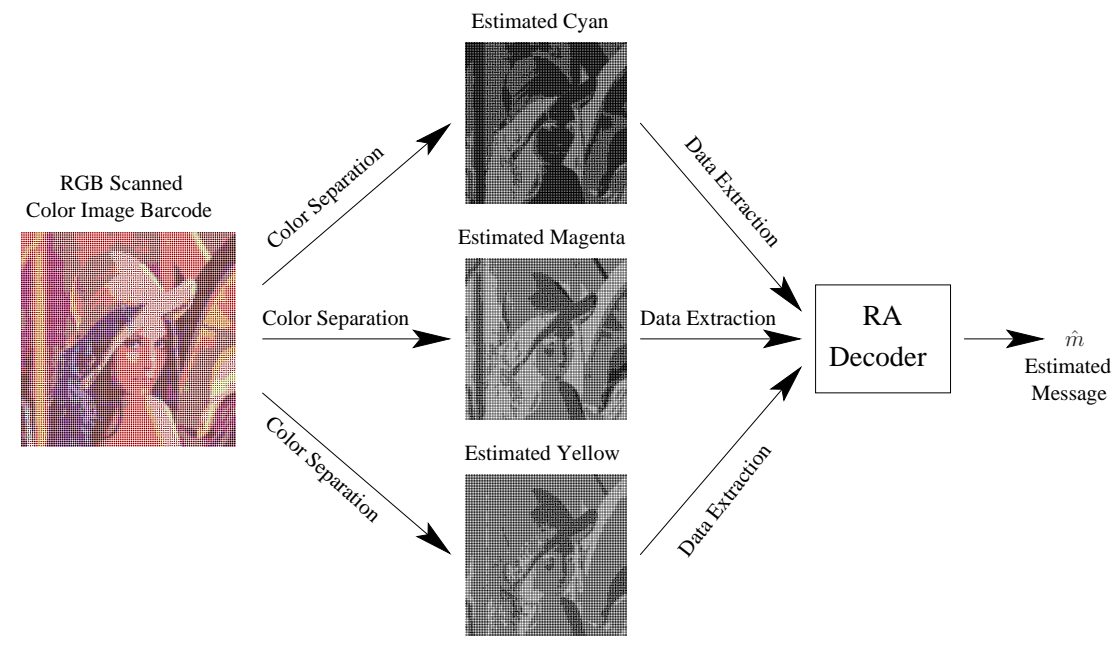

Figure 7. Message recovery from the scanned barcode.

\section{RESULTS}

In the first part of our experiments, we simulated the effect of print-scan channel for a 2400 dpi CMYK color printer and 1200 dpi RGB color scanner combination. Screen frequencies $f_{x}$ and $f_{y}$ were chosen to be 120 cycles per inch (cpi). We simulated four high capacity image barcodes generated by the proposed method in 3 in $\times 3$ in format. The images used in our experiments are shown in Fig. 8.

In order to simulate the inter-separation colorant interactions due to print-scan, ${ }^{20}$ we used a transformation that maps binary CMY printer values to contone RGB scanner values. For this purpose, we measured RGB values of each Neugebauer primary ${ }^{18}$ for the printer from printed targets. Our experimental setup utilized an electrophotographic CMYK color printer (HP Color LaserJet 4650) and a desktop RGB scanner (Epson Perfection 1200S). Average RGB values of 8 Neugebauer primaries were measured from $0.5 \times 0.5$ square-inch patches and stored in a Look-Up Table. The CMY values of each pixel in the color halftone $I_{C M Y}^{b}(x, y)$ image are mapped to RGB scanner values using this table. The effect of physical dot gain is simulated by a spatial blur $^{21}$ on the individual RGB channels of the resulting image. For this purpose, a $7 \times 7$ Gaussian low-pass filter (DC response normalized to 1 ) with variance approximately equal to 0.96 was employed. Finally, $I_{C M Y}^{s}(x, y)$ is obtained by downsampling the individual RGB colorant channels of the resulting image by a factor of 2 .

The embedded data is first extracted from the individual RGB colorant separations. The extracted data is then fed into the RA decoder to estimate the transmitted message. The bit error rates (BER) for RA code rate $1 / 2$ for various threshold values are listed in Table 1 . In the table, the highest bit rate is obtained when the threshold value $(t)$ is set to 0 which corresponds to binary symmetric channel (BSC) model. As the transition is made from BSC to erasure channel model, lower bit error rates can be obtained. Based on the BER in the table for various threshold values, we set threshold value to 0.6 and employ RA codes with a rate of $1 / 3$ in order to achieve error free detection. In this case, we successfully transmit 1800 bytes per inch square area for all images.

In the second part, we tested the proposed image barcodes on a physical (rather than simulated) printer and 


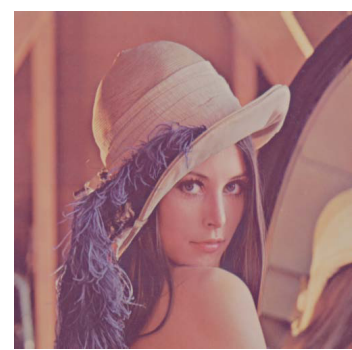

(a) Lena

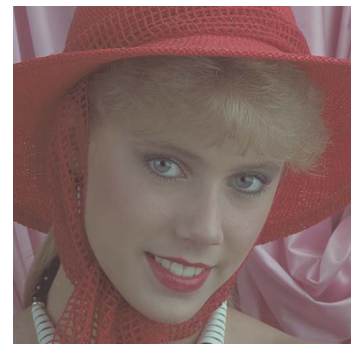

(c) Woman I

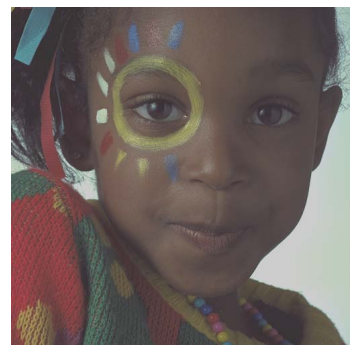

(b) Girl

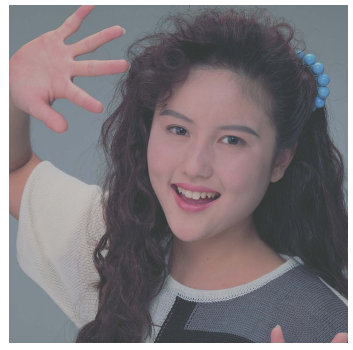

(d) Woman II

Figure 8. Images utilized in our experiments.

\begin{tabular}{c|c|c|c|c} 
& Lena & Girl & Woman I & Woman II \\
\hline \hline$t=0$ & 0.0788 & 0.0618 & 0.0816 & 0.0243 \\
\hline$t=0.2$ & 0.0344 & 0.0346 & 0.0631 & 0.0053 \\
\hline$t=0.4$ & 0.0064 & 0.0247 & 0.0409 & 0.0018 \\
\hline$t=0.6$ & 0.0020 & 0.0480 & 0.0256 & 0.0235 \\
\hline$t=0.8$ & 0.0144 & 0.0959 & 0.0673 & 0.0540 \\
\hline$t=1$ & 0.0858 & 0.1724 & 0.1288 & 0.0915
\end{tabular}

Table 1. BER performance of the proposed method with a RA code rate $1 / 2$ for various threshold $(t)$ values. Rate $1 / 3$ codes provided error free operation for $t=0.6$.

scanner. For this purpose, we first generated image barcodes with screen frequencies $f_{x}$ and $f_{y} 50$ cycles per inch (cpi) $)^{\ddagger}$ for the images shown in Fig. 8 and printed them on HP Color LaserJet 4650. The printed barcodes are then scanned on HP ScanJet 8270 at 1200 dpi resolution. We then separated the individual color channels of the scanned barcode and calculated the BER at each color channel. The observed BER's are reported in Table 2. For all images, the BER observed in blue channel is significantly higher than the BERs observed for the red and green channels. The reason for high BER in blue channel is the color interference between print colorant channels and scanner sensor channels, which is shown to be more significant in blue scanner channel. ${ }^{6}$ Overall, the error rates, though higher than the simulations, are reasonably low and can be compensated for by the use of error correction coding, while maintaining high effective operational, i.e. error-free, data rates. The threshold value $t$ at erasure decoding of error correction coding (in Sec. 3.3) can be selected conveniently for individual color channels based on the color intereference observed in scanner sensor channels.

\section{CONCLUSION}

In this paper, we present a high capacity image barcode scheme for applications that require both high capacity and pleasing visual appearance of the encoded region. The scheme combines orientation modulation based data

\footnotetext{
${ }^{\ddagger}$ The driver through which we accessed the printer did not allow our bi-level color separations to be printed directly by bypassing printer processing and halftoning. We were therefore constrained to working with lower screen frequencies where the impact of the artifacts introduced by the conversions and re-halftoning were minimized.
} 


\begin{tabular}{c|c|c|c} 
& $\begin{array}{c}\text { BER } \\
\text { Red Channel }\end{array}$ & $\begin{array}{c}\text { BER } \\
\text { Green Channel }\end{array}$ & $\begin{array}{c}\text { BER } \\
\text { Blue Channel }\end{array}$ \\
\hline Lena & 0.0002 & 0.0314 & 0.1198 \\
\hline Girl & 0.0108 & 0.048 & 0.185 \\
\hline Woman I & 0.0010 & 0.045 & 0.188 \\
\hline Woman II & 0.027 & 0.018 & 0.129
\end{tabular}

Table 2. BER performance of the proposed image barcodes printed on HP Color LaserJet 4650.

encoding on per-channel basis and color separation. We demonstrate that significant performance improvements can be obtained in terms of embedding rates by sacrificing image fidelity in favor of embedding robustness. Our simulation and experimental results indicate that dot orientation modulation based data embedding can achieve high embedding rates and well suited for per-colorant channel based data encoding in printed documents.

\section{REFERENCES}

1. "Data Matrix barcode FAQ \& tutorial," accessed Jan 2008. [Online]. Available: http://idautomation.com/datamatrixfaq.html

2. R. Villán, S. Voloshynovskiy, O. Koval, and T. Pun, "Multilevel 2D bar codes: Towards high capacity storage modules for multimedia security and management," IEEE Transactions on Information Forensics and Security, vol. 1, no. 4, pp. 405-420, December 2006. [Online]. Available: http://vision.unige.ch/publications/postscript/2006/TIFSmultilevel-2D-barcodes.pdf

3. D. Parikh and G. Jancke, "Localization and Segmentation of A 2D High Capacity Color Barcode," Applications of Computer Vision, 2008. WACV 2008. IEEE Workshop on, pp. 1-6, 2008.

4. O. Bulan, V. Monga, and G. Sharma, "High capacity color barcodes using dot orientation and color separability," in Proc. SPIE: Media Forensics and Security XI, E. J. D. III, J. Dittmann, N. D. Memon, and P. W. Wong, Eds., vol. 7254, Jan. 2009, pp. 725 417-1-7.

5. "Colorzip," accessed Feb. 2010. [Online]. Available: http://www.colorzip.co.jp/en/

6. O. Bulan and G. Sharma, "High capacity color barcodes: Per channel data encoding via orientation modulation in elliptical dot arrays," IEEE Trans. Image Proc., to appear, accepted for publication October 2010.

7. J. Mayer, J. Bermudez, A. Legg, B. Uchoa-Filho, D. Mukherjee, A. Said, R. Samadani, and S. Simske, "Design of high capacity $3 \mathrm{D}$ print codes aiming for robustness to the PS channel and external distortions," in Proc. IEEE Intl. Conf. Image Proc., Nov. 2009, pp. 105-108.

8. S. J. Simske, J. S. Aronoff, and M. Sturgill, "Revenge of the physical - mobile color barcode solutions to security challenges," in Proc. Optical Document Security, San Francisco, 20-22 Jan. 2010, also available as. ${ }^{22}$

9. D. Kacker and J. P. Allebach, "Joint halftoning and watermarking," Signal Processing, no. 4, pp. 1054-1068, Apr. 2003.

10. O. Bulan, V. Monga, G. Sharma, and B. Oztan, "Data embedding in hardcopy images via halftone-dot orientation modulation," in Proc. SPIE: Security, Forensics, Steganography, and Watermarking of Multimedia Contents X, E. J. Delp, P. W. Wong, J. Dittmann, and N. D. Memon, Eds., vol. 6819, Jan. 2008, pp. 68 190C-1-12.

11. O. Bulan, G. Sharma, and V. Monga, "Orientation modulation for data hiding in clustered-dot halftone prints," IEEE Trans. Image Proc., vol. 19, no. 8, pp. 2070-2084, Aug. 2010.

12. D. Shaked, A. Levy, Z. Baharav, and J. Yen, "A Visually Significant Two Dimensional Barcode," Hewlett-Packard Lab., Tech. Rep. HPL-2000-164, 14 Dec. 2001. [Online]. Available: http://www.hpl.hp.com/techreports/2000/HPL2000-164R1.pdf

13. N. Damera-Venkata, J. Yen, V. Monga, and B. Evans, "Hardcopy image barcodes via block-error diffusion," IEEE Trans. Image Proc., vol. 14, no. 12, pp. 1977-1989, Dec. 2005.

14. D. L. Hecht, "Embedded data glyph technology for hardcopy digital documents," in Proc. SPIE: Color hard copy and graphic arts III, J. Bares, Ed., vol. 2171, Mar. 2001, pp. 341-352.

15. C. M. Hains, S. Wang, and K. T. Knox, "Digital color halftones," in Digital Color Imaging Handbook, G. Sharma, Ed. Boca Raton, FL: CRC Press, 2003, chapter 6.

16. P. Roetling, R. P. Loce, and Y. Lin, "Digital halftoning for printing and display of electronic images," in Electronic Imaging Technology, E. R. Dougherty, Ed. Belligham, WA: SPIE, 1999, chapter 7.

17. G. Sharma and H. J. Trussell, "Digital color imaging," IEEE Trans. Image Proc., vol. 6, no. 7, pp. 901-932, Jul. 1997. [Online]. Available: http://www.ece.rochester.edu/ gsharma/papers/dciip97.pdf

18. G. Sharma, "Color fundamentals for digital imaging," in Digital Color Imaging Handbook, G. Sharma, Ed. Boca Raton, FL: CRC Press, 2003, chapter 1. 
19. D. Divsalar, H. Jin, and R. J. McEliece, "Coding theorems for "turbo-like" codes," in Proc. Allerton Conference, Monticello, IL, USA, Sept. 1998, pp. 201-210.

20. J. A. C. Yule, Principles of color reproduction, applied to photomechanical reproduction, color photography, and the ink, paper, and other related Industries. New York: Wiley, 1967.

21. S. Gustavson, "Color gamut of halftone reproduction," J. Imaging Sci. and Tech., vol. 41, no. 3, pp. 283-290, May/Jun. 1997.

22. S. J. Simske, J. S. Aronoff, and M. Sturgill, "Revenge of the physical-mobile color barcode solutions to security challenges," Hewlett-Packard Lab., Tech. Rep. HPL-2010-7, 21 Jan. 2010. [Online]. Available: http://www.hpl.hp.com/techreports/2010/HPL-2010-7.pdf

23. G. Sharma, Ed., Digital Color Imaging Handbook. Boca Raton, FL: CRC Press, 2003. 